\title{
Validación de un instrumento para medir la adherencia al tratamiento en hipertensión y diabetes
}

Validation of an instrument to measure treatment adherence in hypertension and diabetes

\author{
Validação de um instrumento para medir a adesão ao tratamento em \\ hipertensão e diabetes
}

Como citar este artículo:

Romero Guevara Sandra Lucrecia, Parra Dora Inés, Roa Díaz Zayne Milena, Rojas Lyda Z. Validación de un instrumento para medir la adherencia al tratamiento en hipertensión y diabetes. Revista Cuidarte. 2020;11(3):e1062.

http://dx.doi.org/10.15649/cuidarte.1062

Revista Cuidarte

Rev Cuid. 2020; 11(2): e1062

doij http://dx.doi.org/10.15649/cuidarte.1062

E-ISSN: 2346-3414

(1) Sandra Lucrecia Romero Guevara' (1) Dora Inés Parra²

(1) Zayne Milena Roa Díaz ${ }^{3}$

(1) Lyda Z. Rojas ${ }^{4}$

1 Universidad Industrial de Santander, Facultad de Salud, Escuela de Enfermería. Bucaramanga, Colombia. Email: salurome@uis.edu.co Autor de Correspondencia.

2 Universidad Industrial de Santander, Facultad de Salud, Escuela de Enfermería. Bucaramanga, Colombia. Email: doiparra@uis.edu.co

3 Universidad Industrial de Santander, Facultad de Salud, Escuela de Enfermería. Bucaramanga, Colombia. University of Bern, Institute of Social and Preventive Medicine (ISPM), Bern, Switzerland. Email: zayne. roadiaz@ispm.unibe.ch

4 Universidad Industrial de Santander, Facultad de Salud, Escuela de Enfermería. Bucaramanga, Colombia. Centro de Investigación, Fundación Cardiovascular de Colombia, Floridablanca, Colombia. Email:

lyda.rojas@correo.uis.edu.co lydarojas@fcv.org

\section{Resumen}

Introducción: La hipertensión y la diabetes mellitus son enfermedades crónicas que representan una importante carga tanto económica como social. El manejo de estas patologías requiere de estrategias que involucran cambios en el estilo de vida, cumplimiento del tratamiento farmacológico y monitoreo eficaz de la enfermedad. Usualmente, la medición del cumplimiento se enfoca en el tratamiento farmacológico, dejando de lado el enfoque integral que involucra diferentes recomendaciones que son clave para el adecuado control de estas enfermedades. Objetivo: Determinar la validez de constructo y reproducibilidad de la etiqueta de resultado de enfermería "Conducta terapéutica: enfermedad o lesión (1609)" para medir la adherencia al régimen terapéutico en personas con hipertensión arterial y diabetes mellitus tipo 2. Materiales y Métodos: Estudio de corte transversal realizado en 500 usuarios de programas de riesgo cardiovascular en Bucaramanga-Colombia. Se diseñó un instrumento para medir la adherencia al tratamiento con base en la taxonomía Clasificación de Resultados de Enfermería, recomendaciones vigentes de la literatura científica y la experiencia de los investigadores. La validez de constructo fue evaluada a través de un análisis factorial de componentes principales y bajo la metodología Rasch. La reproducibilidad por medio del coeficiente de correlación intraclase en 100 usuarios. Resultados: Se obtuvo un instrumento de 13 ítems que representan 5 indicadores de la etiqueta de resultado de enfermería 1609, los cuales explicaron el $67.62 \%$ de la variación total y se ajustaron al modelo Rasch (unidimensionalidad del constructo adherencia). La reproducibilidad fue del 0.63 (IC 95\% 0.46 - 0.75). Discusión y Conclusiones: Se encontró evidencia de la validez de constructo del instrumento que operacionaliza la etiqueta de resultado de enfermería (1609) para la medición de la adherencia al régimen terapéutico en pacientes con hipertensión y diabetes.

Palabras clave: Estudios de Validación; Encuestas y Cuestionarios; Terminología Normalizada de Enfermería; Cumplimiento y Adherencia al Tratamiento; Hipertensión; Diabetes Mellitus Tipo 2.
Recibido: octubre 12 de 2019

Aceptado: mayo 22 de 2020

Publicado: septiembre 1 de 2020 $\square *$ Correspondencia

Sandra Lucrecia Romero Guevara

E-mail: salurome@uis.edu.co 


\section{Validation of an instrument to measure treatment adherence in hypertension and diabetes}

\section{Abstract}

Introduction: Hypertension and diabetes mellitus are chronic diseases imposing a significant economic and social burden. Managing these diseases requires lifestyle changes, adherence to drug treatment and effective disease monitoring. Measuring compliance usually focuses on drug treatment, leaving aside the comprehensive approach which involves different key recommendations for appropriate control of these diseases. Objective: To determine the construct and reproducibility validity of the Nursing Outcomes Classification label "Therapeutic behavior: illness or injury (1609)" to measure adherence to the therapeutic regimen in people with hypertension and type 2 diabetes mellitus. Materials and Methods: A cross-sectional study was conducted among 500 users of cardiovascular risk reduction programs in Bucaramanga, Colombia. An instrument was designed to measure adherence to treatment based on the Nursing Outcomes Classification taxonomy, current recommendations from scientific literature and researchers experience. Construct validity was assessed using a principal component factor analysis based on the Rasch model. Reproducibility was validated using the intraclass correlation coefficient in 100 users. Results: A 13-item instrument comprising 5 indicators of the Nursing Outcome Classification label (1609) was developed, which explained $67.62 \%$ of the total variation, fitting the Rasch model (unidimensionality of the adherence construct). Reproducibility was 0.63 (95\% Cl 0.46-0.75). Conclusions: Evidence was found for the construct validity of the instrument operationalizing the Nursing Outcomes Classification label (1906) for the measurement of adherence to the therapeutic regimen in patients with hypertension and diabetes.

Key words: Validation Studies; Surveys and Questionnaires; Standardized Nursing Terminology; Treatment Adherence and Compliance; Hypertension; Diabetes Mellitus, Type 2.

\section{Validação de um instrumento para medir a adesão ao tratamento em hipertensão e diabetes}

\section{Resumo}

Introdução: Hipertensão arterial e diabetes mellitus são doenças crônicas que representam uma carga econômica e social significativa. A gestão das doenças requer estratégias que envolvam mudanças no estilo de vida, adesão ao tratamento medicamentoso e monitoramento eficaz das doenças. Normalmente, a medição da conformidade se concentra no tratamento medicamentoso, deixando de lado a abordagem integral que envolve diferentes recomendações que são fundamentais para o controle adequado dessas doenças. Objetivo: Determinar a validade da construção e reprodutibilidade da etiqueta de Classificação dos Resultados de Enfermagem "Conduta terapêutica: doença ou lesão (1609)" para medir a adesão ao regime terapêutico de pessoas com pressão alta e diabetes mellitus tipo 2. Materiais e Métodos: Estudo transversal realizado entre 500 usuários de programas de risco cardiovascular em Bucaramanga-Colômbia. Um instrumento foi projetado para medir a adesão ao tratamento com base na taxonomia da Classificação dos Resultados de Enfermagem, recomendações atuais da literatura científica e experiência dos pesquisadores. A validade da construção foi avaliada através de uma análise dos principais fatores componentes de acordo com a metodologia Rasch. A reprodutibilidade foi validada por meio do coeficiente de correlação intraclasse em 100 usuários. Resultados: Foi obtido um instrumento de 13 itens contendo 5 indicadores da Etiqueta de Resultados de Enfermagem 1609, que explicou $67,62 \%$ da variação total e se encaixa no modelo Rasch (unidimensionalidade da construção de adesão). A reprodutibilidade foi de 0,63 (IC de 95\% 0,46 - 0,75). Conclusões: Encontramos evidências da validade da construção do instrumento que operacionaliza a etiqueta de Classificação dos Resultados de Enfermagem (1609) para medir a adesão ao regime terapêutico de pacientes com hipertensão e diabetes.

Palavras chave: Estudos de Validação; Inquéritos e Questionários; Terminologia Padronizada em Enfermagem; Cooperação e Adesão ao Tratamento; Hipertensão; Diabetes Mellitus Tipo 2. 


\section{Introducción}

La hipertensión arterial (HTA) y la diabetes mellitus (DM) son enfermedades crónicas que representan una importante carga de enfermedad'. La HTA es el principal factor de riesgo de la enfermedad cardiovascular (ECV), afecta a más de mil millones de personas y causa más de diez millones de muertes al año en el mundo. A pesar de los avances en el tratamiento la prevalencia sigue en aumento, se estima una prevalencia global de HTA entre el 30 al $45 \%{ }^{2}$ con un marcado aumento en países de bajos y medianos ingresos ${ }^{3}$. En cuanto a la DM, se considera que existen alrededor de 463 millones de adultos entre 20 y 79 años con diabetes, lo cual corresponde al $9,3 \%$ de la población mundial ${ }^{4}$, en estos se considera que la mortalidad es de aproximadamente el doble en comparación con los pacientes no diabéticos ${ }^{5,6}$.

Tanto la HTA como la DM son enfermedades no curables, la falta de control de las cifras tensionales y de glucosa favorecen el desarrollo de complicaciones que representan una alta carga económica y social ${ }^{7,8}$. Para 2019 la Federación Internacional de Diabetes estimó una mortalidad de 4,2 millones de adultos entre 20 y 79 años y un gasto mundial total directo de 760 mil millones de USD como resultado de la diabetes y sus complicaciones ${ }^{4}$. En cuanto a la hipertensión, esta estuvo relacionada con más de 10 millones de muertes en el 2015 y con un costo mundial directo de más de 370 mil millones de dólares en el 20099,10.

El manejo de estas patologías requiere cambios en el estilo de vida, cumplimiento del tratamiento farmacológico y monitoreo eficaz. Por lo tanto, personas con HTA y DM deben ser adherentes a recomendaciones que incluyen: la restricción de sal y azúcares, el alto consumo de frutas y verduras, la dieta baja en grasa y carbohidratos, la reducción de peso, la actividad física regular, la toma de medicamentos, la restricción en la ingesta de alcohol y el cese del consumo de tabaco, lo cual es esencial para prevenir las complicaciones a largo plazo 2,11,12.

La adherencia al tratamiento ha sido definida por la Organización Mundial de la Salud (OMS) como "El grado en que el comportamiento de una persona - tomar el medicamento, seguir un régimen alimentario y ejecutar cambios del modo de vida - se corresponde con las recomendaciones acordadas de un prestador de asistencia sanitaria"13. La prevalencia de adherencia estimada en enfermedades crónicas es aproximadamente del $50 \%$ en países desarrollados y podría ser menor en países en vía de desarrollo ${ }^{13}$. Las cifras son variables, la prevalencias de adherencia a la medicación en pacientes con HTA oscila entre $24.1 \%$ a $92.7 \%{ }^{14,15}$ y en personas con DM entre el 38.5 y $93.1 \%{ }^{8,16}$.

En cuento a la medición de la adherencia, existen métodos directos e indirectos. Los métodos directos son objetivos, específicos y consisten en determinar en fluidos orgánicos del paciente los niveles del medicamento, algún metabolito o marcador incorporado, sin embargo, son costosos, sofisticados y fuera del alcance de la atención primaria. Los métodos indirectos son sencillos y económicos, por lo que son los más usados en la práctica clínica, pero tienen el inconveniente de ser menos objetivos, debido a que la información obtenida es proporcionada por los pacientes, familiares o el personal de salud, luego tienden a sobreestimar el cumplimiento ${ }^{17-19}$.

A pesar del desarrollo y puesta a prueba de una gran variedad de intervenciones para mejorar la adherencia al régimen terapéutico en enfermedades crónicas y específicamente en HTA y DM, no se ha logrado la eficacia esperada y los resultados son controvertidos; un aspecto clave en la ecuación y que no se le ha dado la importancia necesaria, es la incapacidad de estandarizar métodos eficaces y de fácil aplicación para la medición de la adherencia, usualmente, 
la medición del cumplimiento se enfoca en el tratamiento farmacológico, dejando de lado el enfoque integral de las recomendaciones para un adecuado control de las enfermedades crónicas ${ }^{17-18}$.

En otras palabras, adherirse al régimen terapéutico va mucho más allá de tomarse los medicamentos y requiere de cambios en el estilo de vida para lograr la puesta en práctica de actividades donde los individuos sean conscientes de su papel en el mantenimiento de su propia vida, salud y bienestar ${ }^{20}$. En este sentido, enfermería reconoce su responsabilidad en la comprensión del fenómeno de estudio y la necesidad de la puesta en marcha de soluciones efectivas con base en el soporte teórico que fundamenta y orienta el quehacer de la profesión. Entre las principales teoristas está Dorothea E. Orem quien centra su teoría en el autocuidado y reconoce a la persona como un sujeto activo, con el potencial de aprender a cuidarse y a enfermería como la responsable de ayudar a las personas a alcanzar el conocimiento, la habilidad y motivación necesaria para lograrlo ${ }^{21}$. En este proceso es clave poder identificar y reconocer el déficit de autocuidado en las personas con procesos crónicos de enfermedad que no logran adherirse al régimen terapéutico ${ }^{20}$.

De otra parte, la profesión de enfermería hace uso de herramientas como el proceso de atención de enfermería, que le permite estructurar el cuidado que brinda ${ }^{22}$. Asimismo, cuenta con taxonomías como NANDA Internacional (NANDA-I) ${ }^{23}$, la Clasificación de Intervenciones de Enfermería (CIE) ${ }^{24}$ y la Clasificación de Resultados de Enfermería (CRE) que estandarizan el lenguaje enfermero, específicamente este último contiene etiquetas de resultado que miden y siguen el estado o nivel de salud de un paciente, permitiendo evaluar la eficacia de las intervenciones de enfermería ${ }^{25}$. Evaluar los resultados de enfermería implica seleccionar los mejores indicadores de resultado que se utilizarán, así como la mejor forma y frecuencia para medirlos, adicionalmente, se requiere considerar las necesidades específicas de la población donde se pretende aplicar la etiqueta CRE. Por ejemplo, para medir la adherencia en personas con enfermedades como la HTA y DM, se requiere considerar indicadores tanto del cumplimiento farmacológico, como de comportamientos de autocuidado ${ }^{26,27}$.

El CRE en su $4^{\circ}$ edición incluyó la etiqueta de resultado de enfermería "Conducta Terapéutica: Enfermedad o Lesión (Código 1609)", definida como "acciones personales para paliar o eliminar una patología" del dominio conocimiento y conducta (IV), esta constaba de 17 indicadores medidos a través de una escala Likert de 5 puntos, los cuales involucran aspectos que permiten la medición del grado de cumplimiento de las recomendaciones tanto farmacológicas como las de comportamientos de autocuidado ${ }^{28}$, por tanto, es muy adecuada para medir la adherencia al régimen terapéutico. Dicha etiqueta había sido evaluada previamente para medir la adherencia terapéutica y demostró validez de constructo de 8 de sus indicadores en un estudio en 166 personas con $\mathrm{HTA}^{29}$.

En consideración con lo planteado, el presente trabajo tiene como objetivo determinar la validez de constructo y reproducibilidad de la etiqueta de resultado de enfermería "Conducta Terapéutica: Enfermedad o Lesión (Código 1609)" como instrumento para medir la adherencia al 
régimen terapéutico en personas con HTA y DM2, involucrando tanto lo farmacológico como los cambios en los comportamientos de autocuidado. Lo anterior, con base en los conocimientos teóricos y prácticos de enfermería como ciencia del cuidado y la literatura científica vigente sobre el manejo de la HTA y DM2. Todo esto, para contribuir en la búsqueda de respuestas y soluciones a la problemática de la falta de adherencia al régimen terapéutico en enfermedades crónicas.

\section{Materiales y métodos}

Estudio de corte transversal para la validez de constructo y reproducibilidad de un instrumento para la medición de la adherencia al régimen terapéutico en personas con HTA y DM2, en usuarios del programa de riesgo cardiovascular (RCV) de dos instituciones de atención primaria de la ciudad de Bucaramanga-Colombia. El estudio se llevó a cabo en el periodo comprendido entre los años 2013 y 2014 e hizo parte del proyecto: "Factores asociados a la adherencia al tratamiento farmacológico y no farmacológico en personas con HTA y DM tipo II".

El instrumento sobre el cual realiza la validación se elaboró a partir de la etiqueta de resultado de enfermería "Conducta Terapéutica: Enfermedad o Lesión (Código 1609)"28, dicho instrumento consta de 11 indicadores y 85 ítems, medidos de forma dicotómica y puede verse en detalle en el anexo1. El proceso de construcción, la validez de contenido y facial no se presentan en el presente artículo, al no ser el objetivo del mismo; sin embargo puede ser revisado en el anexo 2.

El estudio incluyó pacientes mayores de 18 años con diagnóstico médico de HTA y DM2, totalmente independientes en su autocuidado y que se encontraran activos (asistencia a control en los últimos 6 meses) en el programa de riesgo cardiovascular de dos instituciones de salud de Bucaramanga. Fueron excluidas gestantes, personas con alteración de la esfera mental y con enfermedades crónicas o severas que limitaran la comunicación.

El cálculo del tamaño de muestra fue estimado para alcanzar una separación y estabilidad de los ítems de $\pm 1 / 2$ lógito con un intervalo de confianza del $99 \%$, resultando en una muestra mínima de 250 personas, sin embargo con el propósito de realizar un análisis por subgrupos (sexo y edad) fueron reclutados un total de 500 sujetos $^{30}$. La muestra fue seleccionada de forma probabilística, por medio de un muestreo aleatorio simple a través de números aleatorios generados en el software OpenEpi. Para la evaluar la reproducibilidad del instrumento se seleccionó aleatoriamente a 100 pacientes de los 500 y pasada una semana se repitió la aplicación del instrumento, manteniendo las mismas condiciones de la primera medida.

Para la recolección de datos de realizó verificación por historia clínica y llamada telefónica de criterios de inclusión y exclusión, se generó un listado de marco muestral para la selección aleatoria de los pacientes. Una vez seleccionados al azar los 500 pacientes, estos fueron contactados a través de llamada telefónica o visita domiciliaria, donde se brindó información general del proyecto y se les invitó a participar en el estudio. Ellos fueron citados al centro de atención primaria más cercano de su residencia, donde una enfermera profesional previamente capacitada y entrenada les proporcionó información detallada del estudio y los participantes dieron su consentimiento informado por escrito.

El instrumento de medición de adherencia fue aplicado cara a cara por dos enfermeras, quienes aclararon a los participantes que no existían respuestas buenas o malas y lo importante era responder con honestidad. La aplicación del instrumento tomó en promedio 15 minutos. Para 
evaluar la reproducibilidad se seleccionaron aleatoriamente 100 usuarios de los 500, a quienes se les aplicó el instrumento a la semana siguiente por segunda vez, manteniendo condiciones similares a la primera medición.

El análisis estadístico fue descriptivo para las características sociodemográficas de la población de estudio y en términos de la validez de constructo que es el objetivo del presente estudio, se realizó un análisis factorial de componentes principales (AFCP) exploratorio con el fin de seleccionar los indicadores e ítems que mejor definían la adherencia al régimen terapéutico, estableciendo como criterio de extracción autovalores iguales o superiores a la unidad, a través de la prueba de Cattell's (scree plot) ${ }^{31}$. Para identificar las dimensiones del instrumento se utilizó una rotación ortagonal tipo varimax, donde los ítems alcanzaron cargas de saturación iguales o superiores a $0.4^{32}$. Una vez seleccionados los ítems e indicadores que explicaban el mayor porcentaje de variación total, se evaluó la consistencia interna por medio del alfa de Cronbach.

La reproducibilidad del instrumento (prueba-reprueba) fue evaluada por medio del coeficiente de correlación intraclase $(\mathrm{CCl})$ y por último, se pasó a realizar un análisis de validez de constructo bajo el modelo Rasch (Rating Scale Model) evaluando desde los aspectos sustantivo, contenido, generalización y estructural ${ }^{33,34}$, adicionalmente, se realizó mapa de Wright persona - ítem; las medidas de ajuste (fit) valoradas fueron los outfit y los infit en términos de errores cuadráticos medios o mean square (MNSQ), los cuales se calculan como la diferencia entre lo observado y lo esperado, cuyo rango productivo para la medida es 0.6 a 1.4 con valor ideal de 1, considerando el objetivo de este estudio ${ }^{35}$. Todos los análisis fueron realizados en el software estadístico Stata v14.0 y Winsteps 3.80.0.

Este estudio consideró la Resolución 008430 de 1993 del Ministerio de Salud de Colombia, por la cual se establecen las normas científicas, técnicas y administrativas para la investigación en salud $^{36}$. Es así, que se estableció como una investigación sin riesgo y contó con el aval del Comité de Ética en Investigación de una institución de salud con amplio reconocimiento en el área metropolitana de Bucaramanga, según acta 312 de 26-29 noviembre de 2012. Además, se obtuvo el aval de las dos instituciones donde se llevó a cabo la investigación. Igualmente, todos los participantes dieron el consentimiento informado por escrito.

\section{Resultados}

\section{Características de la población de estudio}

La mediana de edad de los participantes fue 68 años (Mínimo=35; Máximo=92) y el 69.0\% ( $n=345)$ eran mujeres. Del total de población, 347 personas tenían HTA (69.4\%), 45 DM2 (9.0\%) y 108 las dos patologías (21.6\%). Otras características de la población ya fueron descritas en otra publicación ${ }^{37}$.

\section{Selección de los ítems e indicadores}

Los ítems con variabilidad igual a cero fueron eliminados (7e, 8a, 8bi, 8ji, 8ki, 9b, 9c, 9d, 9f, 9g, 9i). Luego el AFCP identificó entre 17 y 29 factores que explicaban respectivamente el $64,71 \%$ y $79,83 \%$ de la varianza total, por tanto, se reorientó el análisis indicador por indicador para identificar los ítems que mejor evaluaban cada indicador (factor), teniendo en cuenta correlaciones $\geq 0.4$ y el conocimiento de los investigadores sobre las indicaciones dadas a los pacientes HTA 
y DM2.

Una vez que se identificaron los ítems que mejor evaluaban cada indicador, se realizó AFCP, identificando 5 factores (evaluados por medio de 13 ítems) y un porcentaje de variación total de $67.62 \%$ explicado en la población de estudio, con factores de carga entre 0.74 y 0.92 (Tabla 1). En el cuadro 1 se muestra como el Factor 1 corresponde al indicador "cumple con el régimen de medicación" (160905), el Factor 2 a "sigue la dieta prescrita" (160919), el Factor 3 a "cumple con el nivel de actividades prescritas" (160904), El Factor 4 a "supervisar los efectos terapéuticos" (160908) y el Factor 5 a "evita conductas que potencien la patología" (160906). El cuadro 1 muestra la versión final del instrumento (Cuadro 1).

Tabla 1. Análisis factorial de componentes principales con rotación ortogonal varimax (cargas)

\begin{tabular}{|c|c|c|c|c|c|c|}
\hline \multirow[b]{2}{*}{ Ítem } & \multicolumn{5}{|c|}{ Factores } & \multirow[b]{2}{*}{ Uniqueness } \\
\hline & F. 1 & F. 2 & F. 3 & F. 4 & F. 5 & \\
\hline $\begin{array}{l}\text { 1a. Se toma todos los medicamentos } \\
\text { que le han ordenado? }\end{array}$ & 0.8084 & & & & & 0.3284 \\
\hline $\begin{array}{l}\text { 1b. Se toma todas las dosis de los } \\
\text { medicamentos ordenados? }\end{array}$ & 0.8427 & & & & & 0.2923 \\
\hline $\begin{array}{l}\text { 1c. Se toma los medicamentos en las } \\
\text { horas indicadas? }\end{array}$ & 0.7467 & & & & & 0.4225 \\
\hline $\begin{array}{l}\text { 1d. Cumple con las recomendaciones } \\
\text { dadas para la toma de los } \\
\text { medicamentos en relación con los } \\
\text { alimentos? }\end{array}$ & 0.7869 & & & & & 0.3649 \\
\hline $\begin{array}{l}\text { 2a. Cuándo realiza ejercicio lo realiza } \\
\text { como mínimo de } 30 \text { minutos al día? }\end{array}$ & & & 0.9306 & & & 0.1267 \\
\hline $\begin{array}{l}\text { 2b. Realiza ejercicio por lo menos } 4 \\
\text { veces a la semana? }\end{array}$ & & & 0.9272 & & & 0.1352 \\
\hline 3a. Cumple con la dieta baja en sal? & & 0.7555 & & & & 0.3964 \\
\hline 3b. Cumple con la dieta baja en grasas? & & 0.7671 & & & & 0.3779 \\
\hline $\begin{array}{l}\text { 3c. Cumple con una dieta baja en } \\
\text { azúcares? }\end{array}$ & & 0.7867 & & & & 0.3790 \\
\hline $\begin{array}{l}\text { 4a. Consume más de dos copas de licor } \\
\text { o dos vasos de cerveza o bebidas } \\
\text { alcohólicas al día?*}\end{array}$ & & & & & 0.7617 & 0.4195 \\
\hline 4b. Actualmente fuma?* & & & & & 0.6937 & 0.4932 \\
\hline $\begin{array}{l}\text { 6b. Realiza con frecuencia tomas de la } \\
\text { presión arterial y/o glucometría. }\end{array}$ & & & & 0.8674 & & 0.2397 \\
\hline $\begin{array}{l}\text { 6c. Lleva un registro en casa de los } \\
\text { valores de la presión arterial y/o } \\
\text { glucometría (con fecha y hora) }\end{array}$ & & & & 0.8758 & & 0.2339 \\
\hline
\end{tabular}

* Ítems que califican, al contrario

Fuente: elaboración por los autores
Una vez que se identificaron los ítems que mejor evaluaban cada indicador, se realizó AFCP, identificando 5 factores (evaluados por medio de 13 ítems) total de $67.62 \%$ explicado en la población de estudio, con factores de carga entre 0.74 y 0.92 (Tabla 1). En el cuadro 1 se muestra como el Factor 1 corresponde al indicador "cumple con el régimen de medicación" (160905), el Factor 2 a "sigue la dieta prescrita" (160919), el Factor 3 a "cumple con el nivel de actividades prescritas" (160904), El Factor 4 a "supervisar los efectos terapéuticos" (160908) y el Factor 5 a "evita conductas que potencien la patología" (160906). y un porcentaje de variación 
Cuadro 1. Instrumento final para medir la etiqueta de resultado “Conducta terapéutica: enfermedad o lesión (1609)"

"Conducta terapéutica: enfermedad o lesión" 1609

Las siguientes preguntas hacen referencia a su cumplimiento sobre las recomendaciones del tratamiento para la hipertensión arterial y/o diabetes. No existe respuesta buena o mala, lo importante es que responda con total sinceridad SI o No según sea su comportamiento frente a las indicaciones del tratamiento de su enfermedad.

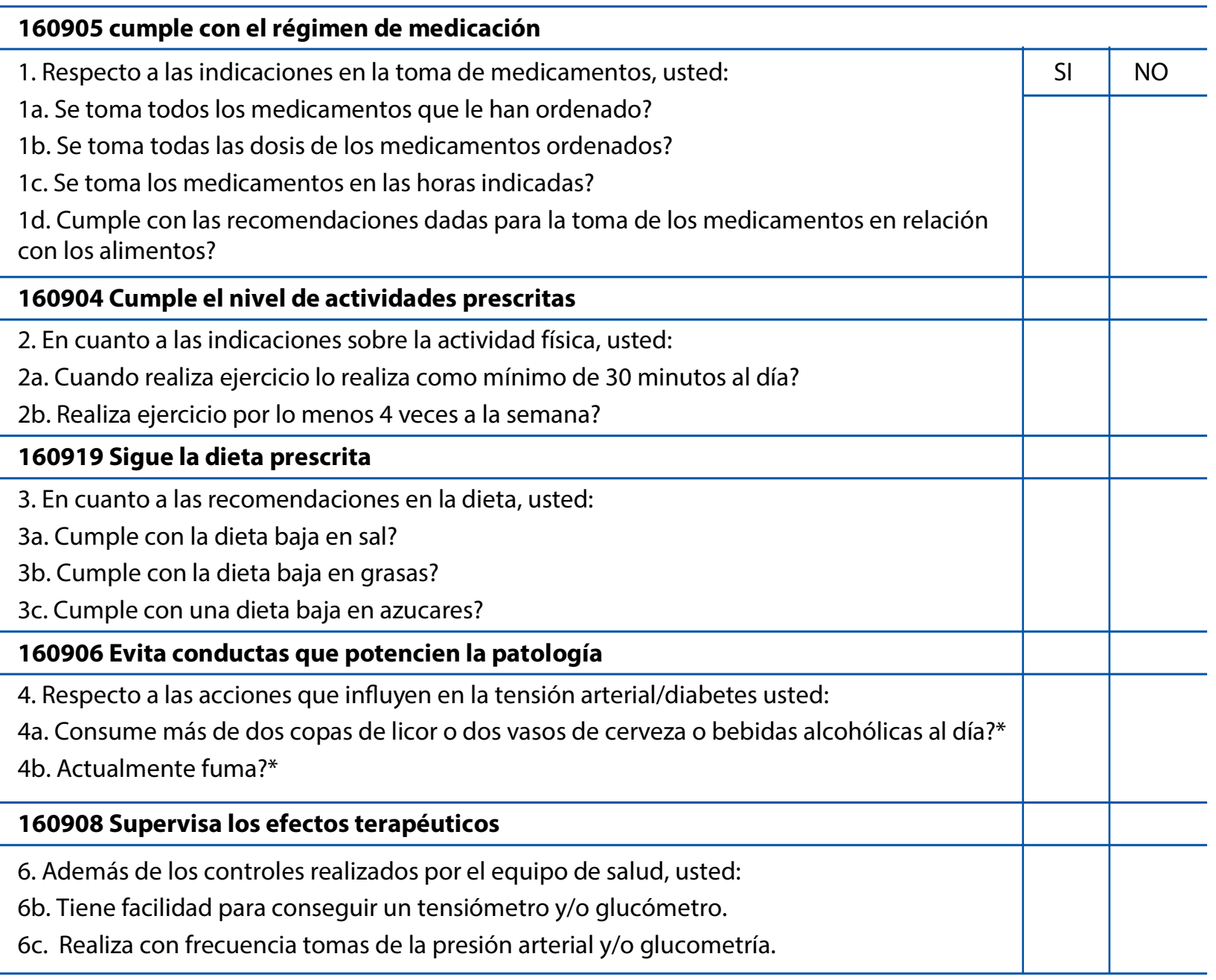

*Items que califican, al contrario

Fuente: elaboración por los autores

\section{Consistencia Interna y Reproducibilidad}

La consistencia global del instrumento fue Cronbach's alphas a=0.61 (IC95\% 0.45-0.77). La reproducibilidad prueba-reprueba reportó un coeficiente de correlación intraclase de 0.63 (IC $95 \% 0.46-0.75)$.

\section{Validez Constructo Rasch}

En primer lugar, se exploró el comportamiento de los estadísticos de separación y confiabilidad, los cuales presentaron valores de 1.11 y 0.55 respectivamente para las personas y de 9.27 y 0.99 para los ítems. Seguidamente se evaluó el aspecto sustantivo de la validez, en donde las dos categorías de respuesta (si y no) cumplieron con criterios de buen funcionamiento, aunque se observaron valores superiores $a \pm 3$ en los outfits estandarizados de diez personas, lo cual conllevo a su eliminación del análisis. 
En relación al aspecto de contenido se evaluó el ajuste de los ítems al modelo por medio de los residuales (infit/outfit), evidenciándose que la mayoría de los ítems tenían valores outfit MNSQ entre 0.5 y 1.5 a excepción del ítem 4a que presentó un valor de 0.12 (Tabla 2). En cuanto al aspecto estructural de la escala a través del análisis de componentes principales de los residuales, se observó que en su conjunto la medida de ítems y personas explicaban el $54.6 \%$ de la varianza bajo el modelo Rasch y el valor del primer contraste fue $9.02 \%$ o 2.6 eigenvalues.

Para definir el puntaje total de adherencia no se consideró apropiado hacer la sumatoria de los puntajes obtenidos en cada indicador para obtener el puntaje global del CRE, esto, teniendo en cuenta que en el proceso se redujo el número de indicadores y por el desbalance entre el número de ítems por cada indicador seleccionado, además, considerando que los 13 ítems responden a la unidimensionalidad del constructo de adherencia, es así que la puntuación del nivel de adherencia iría de 1 a 13, donde 1 corresponde con el nivel mínimo de adherencia y 13 al nivel máximo de adherencia.

De otra parte, aún no está claro en la evidencia el valor o peso que tiene cada uno de los factores (indicadores) en el control tanto de la HTA como la DM, sin embargo, lo que sí está bien definido en las guías de manejo es la importancia de la adherencia a dos estrategias; estas están relacionadas con cambios en el estilo de vida y tratamiento farmacológico, es así, que el cumplimiento al tratamiento farmacológico tiene gran relevancia en especial cuando está presente el riesgo cardiovascular, para nuestro caso el indicador "cumple con el régimen de medicación" quedó representado por el mayor número de ítems (cuatro), seguido de "sigue la dieta prescrita" con tres y los otros indicadores con solo dos ítems.

Tabla 2. Dificultad, error estándar, infit y outfit de los 13 ítems; ordenados por dificultad

\begin{tabular}{ccccccc}
\hline & & \multicolumn{5}{c}{ Medidas de ajuste } \\
\hline \multirow{2}{*}{ Ítem } & Medida & SE & \multicolumn{2}{c}{ Infit } & \multicolumn{2}{c}{ Outfit } \\
\cline { 4 - 7 } & & & MNSQ & ZSTD & MNSQ & ZSTD \\
\hline c1a & -0.83 & 0.16 & 0.92 & -0.7 & 0.80 & -0.7 \\
c1b & -0.04 & 0.13 & 0.98 & -0.2 & 1.13 & 0.7 \\
c1c & -0.41 & 0.14 & 0.95 & -0.6 & 0.93 & -0.2 \\
c1d & -0.88 & 0.16 & 0.84 & -1.5 & 0.55 & -1.8 \\
c2a & 2.03 & 0.11 & 0.98 & -0.3 & 1.05 & 0.5 \\
c2b & 2.58 & 0.11 & 0.89 & -2.1 & 0.89 & -0.9 \\
c3a & -1.37 & 0.19 & 0.99 & 0.0 & 0.90 & -0.2 \\
c3b & -0.85 & 0.16 & 1.02 & 0.2 & 0.83 & -0.6 \\
c3c & -0.45 & 0.15 & 0.99 & -0.1 & 0.97 & 0.0 \\
c4a & -4.95 & 0.74 & 0.79 & -0.1 & 0.12 & -2.9 \\
c4b & -2.85 & 0.32 & 1.34 & 1.2 & 0.74 & -0.4 \\
c6b & 3.00 & 0.12 & 1.14 & 2.3 & 1.49 & 3.3 \\
c6c & 5.00 & 0.18 & 1.16 & 1.3 & 1.13 & 0.5 \\
\hline
\end{tabular}

Medida = Logitos; SE =Standard Error; MNSQ = Mean Square; ZSTD = Standarized

Fuente: elaboración por los autores

Al evaluar la invarianza de los ítems no se encontró comportamiento diferencial por las categorías de sexo y edad. Por último, se elaboró el gráfico de Wright (Figura 1), en el cual se presentan en lógitos la dificultad de los ítems y la habilidad de las personas (en términos de este estudio 
corresponde a la adherencia), observándose que el ítem con mayor grado de dificultad es el $6 c$ con 5 lógitos y los ítems con menor dificultad correspondían al 4a y 4b con -4.95 y -2.85 lógitos. El 90\% ( $n=451)$ personas superó el nivel medio de adherencia medida por el instrumento.

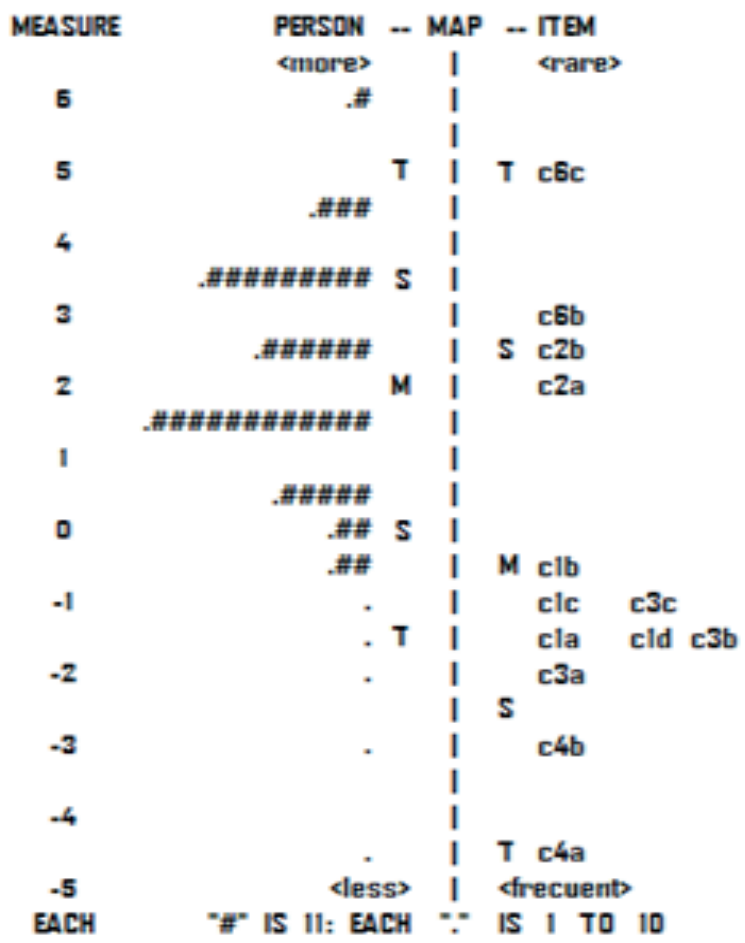

Figura 1. Mapa de habilidad de las personas y dificultad de los 13 ítems en lógitos Fuente: elaboración por los autores

\section{Discusión}

En este estudio, se diseñó y evaluó varios aspectos de la validez de un instrumento para medir la adherencia al tratamiento farmacológico y no farmacológico en pacientes con HTA y DM2 en el ámbito de la atención primaria. Se obtuvo un instrumento conformado por 13 ítems que representan 5 factores, que a su vez corresponden a 5 indicadores de la etiqueta CRE (Cumple con el régimen de medicación, Sigue la dieta prescrita, Cumple el nivel de actividades prescritas, Supervisa los efectos terapéuticos y Evita conductas que potencien la patología); los cuales explican la variación total en un $67.62 \%$ y ajustaron al modelo Rasch reuniendo criterios para respaldar la invarianza y la unidimensionalidad del constructo, lo cual significa que a pesar de que los aspectos evaluados pertenecen a diferentes factores o aspectos de la vida, sus respuestas están orientadas a una sola construcción, en este caso, la adherencia al régimen terapéutico.

En relación a los factores encontrados, estos reflejan la mayoría de las recomendaciones sugeridas en las diferentes guías de manejo y tratamiento de la HTA y DM2, que en general se centran en dos estrategias de tratamiento; cambios en el estilo de vida y terapia farmacológica. Al observar los ítems de acuerdo a los factores identificados, dos están relacionados con la realización de ejercicio y evalúan la cantidad de tiempo dedicada al ejercicio y la frecuencia semanal. En la actualidad, las guías de manejo clínico recomiendan prescribir de 30 a 60 minutos de ejercicio entre 4-7 días a la semana de intensidad moderada, además de las de actividades diarias de ruti$\mathrm{na}^{2,12,38}$ o 3-4 sesiones por semana de actividad física aeróbica moderada a vigorosa-intensa, con un promedio de duración de 40 minutos por sesión ${ }^{39.40}$. Para personas con DM2 se recomienda actividad física de mínimo 150 min por semana, de intensidad moderada, con entrenamiento 
de fuerza e incremento de la actividad física según tolerancia ${ }^{41}$. La Federación Internacional de Diabetes por su parte, recomienda fomentar actividad física aeróbica moderada (50-70\% de la frecuencia cardíaca máxima) 30-45 minutos en 3-5 días por semana ${ }^{42}$.

De otra parte, tres de los ítems encontrados están relacionados con la dieta y evalúan el consumo de sal, grasas y azúcares. En relación con estos aspectos, en las personas con HTA, se indica una ingesta de sodio de 2000 a 2400 mg por día (5-6 g sal o $87 \mathrm{mmol}$ de sodio). Así mismo, se recomienda el consumo de una dieta con énfasis en frutas, verduras, granos enteros, productos lácteos bajos en grasa y proteínas de fuentes vegetales, además se debe limitar la ingesta de dulces, bebidas azucaradas y carnes rojas ${ }^{38-40,43}$. Tanto en diabéticos como en hipertensos recomiendan tener un peso óptimo, restricción calórica si el índice de masa corporal está aumentado (sobrepeso y obesidad), dieta basada en verduras y ácidos grasos insaturados ${ }^{39,41,43}$. Estos enfoques dietéticos se logran siguiendo planes como la dieta DASH (Dietary Approaches to Stop Hypertension), el patrón alimentario de la USDA (United States Department of Agriculture) o la dieta AHA (American Heart Association) ${ }^{39,40,42}$.

Dos de los trece ítems estuvieron asociados con evitar conductas que potencian la patología, tales como el consumo excesivo de alcohol y fumar tabaco. Tanto en pacientes hipertensos como diabéticos no está prohibido el consumo de alcohol, pero se restringe a un nivel moderado. La recomendación particular para reducir la presión arterial en adultos hipertensos es limitar el consumo de alcohol a 2 bebidas por día, sin exceder los $140 \mathrm{~g}$ para hombres y $80 \mathrm{~g}$ para mujeres por semana $2,38,43$. En cuanto al consumo de tabaco y por el alto riesgo que representa este factor, la recomendación es que los proveedores de la atención en salud evalúen el estado de uso de tabaco de todos los pacientes en cada una de las citas de control, les brinden consejería y alternativas para dejar de fumar ${ }^{38,41,42}$.

El cuarto factor identificado, está relacionado con la supervisión de los efectos terapéuticos, a través de los ítems, control ambulatorio de la presión arterial - glucemia y la evaluación de la frecuencia y registro de tomas de presión arterial - glucometría. Para el caso de las personas con hipertensión, el monitoreo domiciliario es recomendado especialmente en aquellos con comorbilidades, sospecha de no adherencia y efecto demostrado de hipertensión de bata blanca o enmascarada; así mismo, se recomienda el registro de los resultados en un diario ${ }^{38,43}$. En cuanto a las personas con diabetes se recomienda evaluar con frecuencia los niveles de glucemia hasta lograr estabilización de los valores. La intensidad y frecuencia de monitoreo de la glucosa debe ser individualizado para atender a las necesidades específicas de cada persona ${ }^{42}$.

El último factor identificado está relacionado con el cumplimiento del régimen de medicación, el cual es evaluado a través de cuatro ítems, los cuales valoran aspectos como la toma de los medicamentos ordenados, las dosis ordenadas, a las horas indicadas y el cumplimiento de las recomendaciones en relación con los alimentos; los tres primeros aspectos han sido considerados en otros test ampliamente utilizados en la evaluación de la adherencia, sin embargo, el cumplimiento de las recomendaciones en relación con los alimentos no ha sido considerado ${ }^{44}$.

En cuanto a los aspectos psicométricos del instrumento, la varianza total explicada en el análisis de los factores y desde el modelo Rasch (ítems - persona) superó el 50\% ${ }^{45-47}$. La moderada reproducibilidad reportada para el instrumento puede explicarse por la homogeneidad de la muestra evaluada, aspecto que ha sido reportado como causal de bajos valores para este estadístico ${ }^{48}$. De otra parte, los bajos niveles de separación y confiabilidad para las personas, además del cuestionable coeficiente de alpha de Cronbach son indicadores de dificultad en 
la capacidad del instrumento para distinguir entre sujetos con alta y baja adherencia ${ }^{49}$, hallazgos que sugieren la necesidad de incluir más ítems ${ }^{50,51}$. Contrario a lo anterior, los altos valores reportados para confiabilidad y separación en ítems, respaldan la robustez de la muestra para confirmar la jerarquía de dificultad de los mismos ${ }^{52}$.

Al comparar los hallazgos del presente trabajo con el único estudio encontrado sobre validación de la etiqueta CRE Conducta Terapéutica para la medición de la adherencia al régimen terapéutico, se encontró similitud en la invarianza de la medida al comparar por grupos de sexo y en los estadísticos de confiabilidad y separación de los ítems. Asimismo, tanto el indicador como el ítem que involucró el consumo de más de dos copas de licor o dos vasos de cerveza o bebidas alcohólicas al día presentaron los menores nivel de dificultad, en ambos trabajos ${ }^{29}$. En cuanto a diferencias con el estudio en mención, es importante aclarar que ellos no utilizaron la metodología de análisis factorial. En cuanto a metodología Rasch el presente trabajo obtuvo mejor ajuste al modelo, al utilizar una escala de calificación dicotómica, superando el valor reportado tras la recodificación a 3 categorías de respuesta ${ }^{29}$. Asimismo, en el estudio en comparación el ítem que presentó mayor dificultad, fue el relacionado con la supervisión de efectos secundarios al tratamiento y para los sujetos del presente trabajo fue el llevar un registro en casa de los valores obtenidos de la presión arterial y glucometría (fecha, hora y valores) ${ }^{29}$.

En la literatura en salud, existen gran variedad de instrumentos para la medición de la adherencia en enfermedades crónicas, sin embargo, muchos de ellos están enfocados específicamente a lo farmacológico, otros son poco específicos para los procesos de enfermedad o son poco prácticos para la aplicación en atención primaria ${ }^{32}$. Dentro de la gran variedad de instrumentos desarrollados, vale la pena mencionar el instrumento de adherencia al tratamiento en falla cardiaca Achury y col., el cual, al igual que en nuestro caso fue construido a partir de una revisión de la literatura, la experiencia de trabajo con la población y del uso de la taxonomía de enfermería $\mathrm{CRE}^{53}$. Dicho instrumento ha evolucionado y a la fecha ya ha sido validado en enfermedad cardiovascular, contempla 7 dimensiones relacionadas con los aspectos claves en la adherencia al régimen terapéutico (medicamentos, nutrición, actividad física, otros factores de riesgo, seguimiento/control, autoeficacia y aspectos generales), de los cuales 5 son considerados en la versión final de nuestro instrumento y vislumbra la posibilidad de contemplar además de lo mencionado, la autoeficacia como un aspecto clave para el logro de los resultados esperados ${ }^{54}$.

De otra parte, está el cuestionario de Bonilla y Gutiérrez para evaluar la adherencia al tratamiento en pacientes con enfermedad cardiovascular, que ha sido validado en múltiples regiones y grupos poblacionales, cuenta con muy buenas propiedades psicométricas y si bien, no mide la adherencia al régimen terapéutico en sí, resulta de gran valor dado que orienta sobre los factores que llevan a la persona a no ser adherente al régimen terapéutico y a la percepción del riesgo en los comportamientos que adoptan las personas en relación con la salud ${ }^{55}$. Por lo tanto, consideramos que son instrumentos complementarios, ya que uno permite evaluar el nivel de adherencia y el otro identifica los factores o barreras asociados a la misma, desde las expectativas del paciente y específicamente ha sido validado en población con enfermedad cardiovascular ${ }^{56}$.

\section{Limitaciones del estudio}

En cuanto a las limitaciones del estudio es importante aclarar que la etiqueta CRE, Conducta Terapéutica: Enfermedad o Lesión fue retirada desde la $5^{a}$ edición. Igualmente, es importante mencionar que, aunque la muestra fue seleccionada aleatoriamente y se tuvo en cuenta a 
usuarios de programas de RCV de centros de atención tanto del sector público como privado de la ciudad de la ciudad de Bucaramanga-Colombia, es posible que los resultados no sean generalizables para todos los usuarios del país, dado que personas con un nivel educativo o estrato socioeconómico mayor pudieran generar algún cambio en la carga de los ítems. Adicionalmente, las propiedades métricas de sensibilidad al cambio no fueron evaluadas debido al diseño metodológico del presente estudio.

También, es importante que futuros estudios establezcan un periodo de tiempo prudente para evaluar cada uno los ítems, por ejemplo: en la última semana, dos semanas o 4 semanas; así mismo contemplar la inclusión de otros ítems que aporten a la obtención de una clasificación del grado de adherencia y que incluya la dimensión de autoeficacia, como un aspecto clave en la gestión del autocuidado.

\section{Contribuciones al campo de enfermería, salud o política pública}

Teniendo en cuenta que el auto reporte es considerado un método práctico y eficiente para evaluar la adherencia al tratamiento ${ }^{57}$, el grupo de investigación considera que este trabajo logró consolidar una propuesta de instrumento de fácil diligenciamiento, que incluye aspectos claves en la medición de la adherencia y a su vez, es coherente con las directrices de las guías basadas en la evidencia; lo cual permite la planeación y aplicación de intervenciones con enfoque individualizado, adaptadas y ajustadas para dar respuesta a las necesidades reales de la población con enfermedad cardiovascular ${ }^{58}$.

Una fortaleza del estudio, es el uso complementario del análisis factorial de componentes principales y el análisis Rasch para la evaluación de la validez de constructo del instrumento, la mayoría de estudios de validación realizan solo un método y con mayor frecuencia los pertenecientes a la teoría clásica. Es importante señalar que la etiqueta Conducta Terapéutica: enfermedad o lesión es multifactorial y la construcción de la adherencia ha sido evolutiva y permanece sin unificación, un aspecto clave para la construcción de instrumentos válidos para la medición y comparabilidad de estas construcciones. Por tanto, este trabajo se convierte en un incentivo para continuar mejorando y afinando la medición de la adherencia en poblaciones con enfermedades crónicas y en particular en los programas de RCV.

De acuerdo con la revisión de la literatura, es el primer instrumento que evalúa la adherencia tanto del tratamiento farmacológico como no farmacológico en pacientes con HTA y DM2; además visto desde el cumplimiento de las recomendaciones del régimen terapéutico y no desde las barreras de la adherencia, lo cual constituye un aporte en la identificación del déficit de autocuidado y una base para orientar y contribuir en el cuidado de las personas con diabetes e hipertensión; todo esto, sin dejar de reconocer el potencial de autocuidado en cada persona y el rol de enfermería como mediador en el proceso para ayudar a la persona a obtener los conocimientos, desarrollar las habilidades y alcanzar la motivación necesarios para lograr los cambios en los estilos de vida.

\section{Conclusiones}

El análisis psicométrico de la etiqueta CRE Conducta Terapéutica: Enfermedad o Lesión (1609) para medir la adherencia al régimen terapéutico en HTA y DM2, compuesta de 13 ítems (que representan 5 indicadores de dicha etiqueta), explica el $67.62 \%$ de la varianza total, además se aportó evidencias de la invariabilidad y unidimensionalidad del constructo de adherencia al 
régimen terapéutico.

El instrumento diseñado puede ser práctico y útil para medir la adherencia al régimen terapéutico en programas RCV, especialmente pacientes con HTA y DM2, aunque es necesaria la validación en pacientes con otras características, específicamente personas de rango socioeconómico medio-alto y un nivel educativo alto que el de la población de este estudio.

Conflicto de Intereses: Los autores declaran no tener conflicto de intereses.

Financiación: Este trabajo hace parte de la Unión Temporal CARDIECOL, una alianza de varias instituciones educativas y de salud entre ellas la Universidad Industrial de Santander. Financiado por COLCIENCIAS según convocatoria No 998-2014, código 47755.

\section{Referencias}

1. Ning M, Zhang Q, Yang M. Comparison of self-reported and biomedical data on hypertension and diabetes: findings from the China Health and Retirement Longitudinal Study (CHARLS). BMJ Open. 2016;6(1):e009836. http://dx.doi.org/10.1136/bmjopen-2015-009836

2. Williams B, Mancia G, Spiering W, Agabiti Rosei E, Azizi M, Burnier M, et al. 2018 ESC/ESH Guidelines for the management of arterial hypertension. Eur Heart J. 2018;39(33):3021-104. https://doi.org/10.1093/eurheartj/ehy339

3. World Health Organization. World Health Statistics 2017: Monitoring health for the SDGs 2017. http://www.who.int/gho/publications/world_health_statistics/2017/en/

4. International Diabetes Federation. Atlas De La Diabetes De La Fid 9a Edición 20192019. https://www.diabetesatlas.org/es/

5. Xu Z, Yu D, Yin X, Zheng F, Li H. Socioeconomic status is associated with global diabetes prevalence. Oncotarget. 2017;8(27). https://doi.org/10.18632/oncotarget.17902

6. Gerrits EG, Landman GW, Bilo HJ. Mortality Trends in Diabetes Mellitus. JAMA Internal Medicine. 2015;175(3):469-. https://doi.org/10.1001/jamainternmed.2014.7985

7. Bener A, Kim EJ, Mutlu F, Eliyan A, Delghan H, Nofal E, et al. Burden of diabetes mellitus attributable to demographic levels in Qatar: an emerging public health problem. Diabetes Metab Syndr. 2014;8(4):216-20. https://doi.org/10.1016/j.dsx.2014.09.005

8. Chang PY, Chien LN, Lin YF, Chiou HY, Chiu WT. Nonadherence of Oral Antihyperglycemic Medication Will Increase Risk of End-Stage Renal Disease. Medicine (Baltimore). 2015;94(47):e2051. https://doi.org/10.1097/MD.0000000000002051

9. Forouzanfar MH, Liu P, Roth GA, Ng M, Biryukov S, Marczak L, et al. Global Burden of Hypertension and Systolic Blood Pressure of at Least 110 to $115 \mathrm{~mm} \mathrm{Hg}$, 1990-2015. JAMA. 2017;317(2):165-82. https://doi.org/10.1001/jama.2016.19043

10.Frieden TR, Jaffe MG. Saving 100 million lives by improving global treatment of hypertension and reducing cardiovascular disease risk factors. The Journal of Clinical Hypertension. 2018;20(2):208-11. https://doi.org/10.1111/jch.13195

11.Perl S, Niederl E, Kos C, Mrak P, Ederer H, Rakovac I, et al. Randomized Evaluation of the Effectiveness of a Structured Educational Program for Patients With Essential Hypertension. Am J Hypertens. 2016;29(7):866-72. https://doi.org/10.1093/ajh/hpv186

12. The American Diabetes Association. 5. Lifestyle Management: Standards of Medical Care in Diabetes-2019. Diabetes Care. 2019;42(Suppl 1):S46-S60. https://doi.org/10.2337/dc19-S005

13.World Health Organization. Adherencia a los tratamientos a largo plazo: pruebas para la acción: Organización Mundial de la Salud; 2004. http://www.farmacologia.hc.edu.uy/images/WHO-Adherence-Long-Term-Therapies-Spa-2003.pdf 
14.Sandy R, Connor U. Variation in medication adherence across patient behavioral segments: a multi-country study in hypertension. Patient Prefer Adherence. 2015;9:1539-48.

https://doi.org/10.2147/PPA.S91284

15.Tang KL, Quan H, Rabi DM. Measuring medication adherence in patients with incident hypertension: a retrospective cohort study. BMC health services research. 2017;17(1):135. https://doi.org/10.1186/s12913-017-2073-y

16.Krass I, Schieback P, Dhippayom T. Adherence to diabetes medication: a systematic review. Diabetic Medicine. 2014;32(6):725-37. https://doi.org/10.1111/dme.12651

17. Nogués $\mathbf{X}$, Sorli $\mathbf{M}$, Villar J. Instrumentos de medida de adherencia al tratamiento. An Med Interna. 2007;24(3):138-41. https://doi.org/10.4321/S0212-71992007000300009

18.Perez-Escamilla B, Franco-Trigo L, Moullin JC, Martinez-Martinez F, Garcia-Corpas JP. Identification of validated questionnaires to measure adherence to pharmacological antihypertensive treatments. Patient Prefer Adherence. 2015;9:569-78.

https://doi.org/10.2147/PPA.S76139

19.López LA, Romero SL, Parra D I, Rojas LZ. Adherencia al tratamiento: Concepto y medición. Hacia promoc salud. 2016;21(1):117-37. http://dx.doi.org/10.17151/hpsal.2016.21.1.10

20.Olivella-Fernandez MC, Bastidas-Sánchez CV, Castiblanco-Amaya MA, Universidad del T. Adherence to Self-Care among Persons with Cardiovascular Disease: An Approach Based on the Orem Model of Nursing. Aquichan. 2012;12(1):53-61.

https://doi.org/10.5294/aqui.2012.12.1.5

21.Naranjo Hernández Y, Concepción Pacheco JA, Rodríguez Larreynaga M. La teoría Déficit de autocuidado: Dorothea Elizabeth Orem. Gaceta Médica Espirituana.2017;19(3):89-100.

http://scielo.sld.cu/scielo.php?script=sci_arttext\&pid=S1608-89212017000300009\&lng=es\&nrm=iso

22.Reina G NC. El proceso de enfermería: instrumento para el cuidado. Umbral Científico. 2010(17):18-23. https://www.redalyc.org/articulo.oa?id=30421294003

23.Herdman TH, Kamitsuru S, (editors). NANDA International. Diagnósticos enfermeros: Definiciones y clasificación 2018-2020. 11ª ed. ed: Elsevier España; 2018.

24.Butcher HK, Bulechek GM, Dochterman JMM, Wagner CM. Nursing Interventions Classification (NIC). . 7a ed. ed: Elsevier España; 2019. 505 p.

25.Moorhead S, Swanson E, Johnson M, Maas ML. Clasificación de resultados de enfermería (CRE) Medición de Resultados en Salud. 6ª ed. ed: Elsevier España; 2018.

26.Moreira RP, de Araujo TL, Cavalcante TF, Guedes NG, Costa AG, Lopes MV. Validation of the mobility nursing outcome in stroke survivors. Int J Nurs Knowl. 2013;24(3):157-62. https://doi.org/10.1111/j.2047-3095.2013.01245.x.

27.Sousa AG, de Araujo TL, Cavalcante TF, Oliveira MV, Oliveira-Kumakura AR, Chaves FB. Clinical validation of the nursing outcome falls prevention behavior in people with stroke. Appl Nurs Res. 2017;33:67-71. https://doi.org/10.1097/MD.0000000000002051

28.Moorhead S, Johnson M, Meridean M, E S. Clasificación de Resultados de Enfermería (NOC). 4th ed. España: Elsevier 2009.

29.Páez AN, Caballero LP, López N, Oróstegui M, Orozco LC, Valencia LI. Construct validity of an instrument to assess patient adherence to antihypertensive treatment. Enfermería Global. 2014;13(2):48-57. https://doi.org/10.6018/eglobal.13.2.170111

30.Linacre JM. Sample size and item calibration stability. Rasch measurement transactions. 1994;7(4):328. https://www.rasch.org/rmt/rmt74m.htm

31.Guttman L. Some necessary conditions for common-factor analysis. Psychometrika. 1954;19(2):149-61. https://doi.org/10.1007/BF02289162

32.Hair JF. Multivariate Data Analysis: Prentice Hall; 2010.

33.Messick S. Validity of psychological assessment: Validation of inferences from persons' re- 
sponses and performance as scientific inquiry into score meaning. American Psychologist. 1995;50(9):741-9. https://doi.org/10.1037/0003-066X.50.9.741

34.Wolfe EW, Smith EV, Jr. Instrument development tools and activities for measure validation using Rasch models: part II--validation activities. J Appl Meas. 2007;8(2):204-34. Epub 2007/04/19. http://www.ncbi.nlm.nih.gov/pubmed/17440262

35.Orozco LC. Medición en salud: diagnóstico y evaluación de resultados: un manual critico más allá de lo básico: Universidad Industrial de Santander; 2010 2010. 213 p.

36.Ministerio de Salud y Protección Social de Colombia. Política de atención integral en salud "Un sistema de salud al servicio de la gente". In: Imprenta Nacional de Colombia editor. Bogotá D.C.2016. p. 97.

37.Romero SL, Parra DI, Sánchez JM, Rojas LZ. Adherencia terapéutica de pacientes con hipertensión arterial y diabetes mellitus tipo 2 de Bucaramanga, Colombia. Revista de la Universidad Industrial de Santander Salud. 2017;49(1):37-44.

http://dx.doi.org/10.18273/revsal.v49n1-2017004

38.Leung AA, Daskalopoulou SS, Dasgupta K, McBrien K, Butalia S, Zarnke KB, et al. Hypertension Canada's 2017 Guidelines for Diagnosis, Risk Assessment, Prevention, and Treatment of Hypertension in Adults. Can J Cardiol. 2017;33(5):557-76. http://dx.doi.org/10.1016/j.cjca.2017.03.005

39.Eckel RH, Jakicic JM, Ard JD, de Jesus JM, Houston Miller N, Hubbard VS, et al. 2013 AHA/ ACC guideline on lifestyle management to reduce cardiovascular risk: a report of the American College of Cardiology/American Heart Association Task Force on Practice Guidelines. Circulation. 2014;129(25 Suppl 2):S76-99. https://doi.org/10.1161/01.cir.0000437740.48606.d1

40.James PA, Oparil S, Carter BL, Cushman WC, Dennison-Himmelfarb C, Handler J, et al. 2014 evidence-based guideline for the management of high blood pressure in adults: report from the panel members appointed to the Eighth Joint National Committee (JNC 8). JAMA. 2014;311(5):507-20. https://doi.org/10.1001/jama.2013.284427

41.Garber AJ, Abrahamson MJ, Barzilay Jl, Blonde L, Bloomgarden ZT, Bush MA, et al. Consensus Statement by the American Association of Clinical Endocrinologists and American College of Endocrinology on the Comprehensive Type 2 Diabetes Management Algorithm - 2017 Executive Summary. Endocr Pract. 2017;23(2):207-38. Epub 2017/01/18. http://journals.aace.com/doi/pdf/10.4158/EP161682.CS

42.International Diabetes Federation Guideline Development G. Global guideline for type 2 diabetes. Diabetes Res Clin Pract. 2014;104(1):1-52. Epub 2014/02/11. https://doi.org/10.1016/j.diabres.2012.10.001

43.Fox CS, Golden SH, Anderson C, Bray GA, Burke LE, de Boer IH, et al. Update on Prevention of Cardiovascular Disease in Adults With Type 2 Diabetes Mellitus in Light of Recent Evidence: A Scientific Statement From the American Heart Association and the American Diabetes Association. Circulation. 2015;132(8):691-718. https://www.ahajournals.org/doi/pdf/10.1161/cir.0000000000000230

44.Rodríguez MÁ, García-Jiménez $\mathbf{E}$, Amariles $\mathbf{P}$, Rodríguez A, José $\mathbf{M}$. Revisión de tests de medición del cumplimiento terapéutico utilizados en la práctica clínica. Atención Primaria. 2008;40(8):413-8. https://doi.org/10.1157/13125407

45.Wright BD, Linacre JM, Gustafson JE, Martin-Löf P. Reasonable mean-square fit values. Rasch measurement transactions. 1994;8(370). https://www.rasch.org/rmt/rmt83b.htm.

46.Floyd FJ, Widaman KF. Factor analysis in the development and refinement of clinical assessment instruments. Psychological Assessment. 1995;7(3):286-99.

https://doi.org/10.1037/1040-3590.7.3.286

47.Pallant JF, Tennant A. An introduction to the Rasch measurement model: An example us- 
ing the Hospital Anxiety and Depression Scale (HADS). British Journal of Clinical Psychology. 2007;46:1-18. https://doi.org/10.1348/014466506X96931

48.Prieto L, Lamarca R, Casado A. La evaluación de la fiabilidad en las observaciones clínicas: el coeficiente de correlación intraclase. Medicina clínica. 1998;110(4):142-5. https://www.mvclinic.es/wp-content/uploads/Prieto-Coeficiente-correlaci\%C3\%B3n-intraclase.pdf .

49.Lerdal A, Opheim R, Gay CL, Moum B, Fagermoen MS, Kottorp A. Psychometric limitations of the 13-item Sense of Coherence Scale assessed by Rasch analysis. BMC Psychol. 2017;5(1):18. https://doi.org/10.1186/s40359-017-0187-y

50.Nielsen MG, Ornbol E, Vestergaard M, Bech P, Christensen KS. The construct validity of the Major Depression Inventory: A Rasch analysis of a self-rating scale in primary care. J Psychosom Res. 2017;97:70-81. https://doi.org/10.1016/j.jpsychores.2017.04.001

51.DeVon HA, Block ME, Moyle-Wright P, Ernst DM, Hayden SJ, Lazzara DJ, et al. A psychometric toolbox for testing validity and reliability. J Nurs Scholarsh. 2007;39(2):155-64. https://doi.org/10.1111/j.1547-5069.2007.00161.x

52.Linacre JM. Reliability and separation of measures. A user's guide to Winsteps Ministep Rasch-model computer programs (version 3.81. 0). 2014;

http://www.winsteps.com/winman/reliability.htm

53.Achury DM, Rodríguez SM, Sepulveda G, Giraldo IC. Validez y confiabilidad de un instrumento evaluativo de adherencia en pacientes con falla cardiaca. Enfermería Global. 2012;11(2). https://doi.org/10.6018/eglobal.11.2.138461

54.Achury-Beltrán LF. Validez y confiabilidad del cuestionario para medir la adherencia al tratamiento de pacientes con enfermedad cardiovascular. Aquichan. 2017;17(4):460-71. https://doi.org/10.5294/aqui.2017.17.4.9

55.Bonilla Ibáñez CP, Gutiérrez de Reales E. Desarrollo y características psicométricas del instrumento para evaluar los factores que influyen en la adherencia a tratamientos farmacológicos y no farmacológicos en personas con factores de riesgo de enfermedad cardiovascular. Avances en Enfermería. 2014;32:53-62. http://dx.doi.org/10.15446/av.enferm.v32n1.46035

56.Andrade B, Céspedes V. Adherencia al tratamiento en enfermedad cardiovascular: rediseño y validación de un instrumento. Enfermería Universitaria. 2017;14(4):266-76. http://www.sciencedirect.com/science/article/pii/S1665706317300702

57.Osterberg L, Blaschke T. Adherence to medication. N Engl J Med. 2005;353(5):487-97. https://doi.org/10.1056/NEJMra050100

58.Esquivel N, Díaz LP, Cañon W. Tailored interventions in people with cardiovascular disease: towards a nursing approach for individualized care. 2019;10(3). https://doi.org/10.15649/cuidarte.v10i3.947

59.Souza AR, Caldeira S, Prado T, Camargo FA, Campos E. The contribution of the Rasch Model to the clinical validation of nursing diagnoses: integrative literature review. International journal of nursing knowledge. 2016;29(2). https://doi.org/10.1111/2047-3095.12162 


\section{Anexo 1.}

Instrumento inicial para medir la etiqueta de resultado “Conducta terapéutica: enfermedad o lesión (1609)" "CONDUCTA TERAPÉUTICA: ENFERMEDAD O LESIÓN" 1609

Las siguientes preguntas hacen referencia a su cumplimiento sobre las recomendaciones del tratamiento para la hipertensión arterial y/o diabetes. No existe respuesta buena o mala, lo importante es que responda con total sinceridad SI o No según sea su comportamiento frente a las indicaciones del tratamiento de su enfermedad.

\section{5 cumple con el régimen de medicación (4 ítems)}

1. Respecto a las indicaciones en la toma de medicamentos, usted:

1a. Se toma todos los medicamentos que le han ordenado?

1b. Se toma todas las dosis de los medicamentos ordenados?

1c. Se toma los medicamentos en las horas indicadas?

1d. Cumple con las recomendaciones dadas para la toma de los medicamentos en relación con los alimentos?

160904 Cumple el nivel de actividades prescritas (4 ítems)

2. En cuanto a las indicaciones sobre la actividad física, usted:

2a. Cuando realiza ejercicio lo realiza como mínimo de 30 minutos al día?

2b. Realiza ejercicio por lo menos 4 veces a la semana?

2c. Antes de empezar el ejercicio usted realiza calentamiento y estiramiento?

$2 d$. Posterior al ejercicio realiza enfriamiento con estiramiento?

160919 Sigue la dieta prescrita (6 ítems)

3. En cuanto a las recomendaciones en la dieta, usted:

3a. Cumple con la dieta baja en sal?

3b. Cumple con la dieta baja en grasas?

3c. Cumple con una dieta baja en azucares?

3d. Cumple con la dieta baja en harina y almidones?

3e. Cumple con el incremento en el consumo de frutas y verduras?

3f. Evita el consumo de alimentos como: postres, dulces, panes, gaseosas.

160906 Evita conductas que potencien la patología (4 ítems)*

4.Respecto a las acciones que influyen en la tensión arterial/diabetes usted:

4a. Consume más de dos copas de licor o dos vasos de cerveza o bebidas alcohólicas al día?*

4b. Actualmente fuma?*

4c. Ha tenido situaciones que le generen disgusto (rabia) en la última semana?*

4d. Ha tenido situaciones que le generen preocupación en la última semana?*

$\mathbf{1 6 0 9 1 6}$ Solicita cita con profesional sanitario cuando es necesario 160921 Obtiene asesoramiento de un profesional cuando es necesario (5 ítems)

5. ¿Asiste a cita o control con médico o enfermera, cuando?

5a. Asiste a cita o control cuando se siente enfermo

5b. Asiste a cita o control cuando tiene citas programadas

$5 c$. Asiste a cita o control cuando tiene dudas o inquietudes acerca del tratamiento

$5 \mathrm{~d}$. Asiste a cita o control cuando los valores de la tensión arterial o glicemia (azúcar) se encuentran alterados

5e. Asiste a cita o control cuando requiere mostrar resultados de laboratorio

160908 Supervisa los efectos terapéuticos

6. Además de los controles realizados por el equipo de salud, usted:

6a. Tiene facilidad para conseguir un tensiómetro y/o glucómetro

6b. Realiza con frecuencia tomas de la presión arterial y/o glucometría.

$6 c$. Lleva un registro en casa de los valores obtenidos de la presión arterial y/o glucometría (fecha, hora y valores). 
160911 Supervisa los cambios en el estado de enfermedad (9 ítems hipertensión y 7 ítems diabetes)

7. Reconoce los síntomas cuando se le sube la presión arterial? ¿Cuáles?

7a. Alteraciones visuales (ver borroso, luces o manchas oscuras)

7b. Calor

7c. Dolor de cabeza

7d. Palpitaciones

7e. Sangrado nasal

7f. Sentir un pito o zumbido en el oído

7g. Sudoración

7h. Debilidad/cansancio

7i. Enrojecimiento de la cara

\begin{tabular}{|l|l|l|l} 
SI & NO & $\begin{array}{l}\text { 7. Reconoce los síntomas cuando se le sube } \\
\text { el azúcar? Cuáles? }\end{array}$ & SI \\
\cline { 3 - 4 } & & $\begin{array}{l}\text { 7ai. Sed intensa } \\
\text { 7bi. Boca seca } \\
\text { 7ci. Necesidad de orinar en bastante } \\
\text { cantidad } \\
\text { 7di. Debilidad/cansancio } \\
\text { 7ei. Alteraciones visuales } \\
\text { 7fi. Mareo (borrachera) } \\
\text { 7gi. Confusión/Incoherencia (atontado, } \\
\text { embotado) }\end{array}$ & \\
\end{tabular}

160909 Supervisa los efectos secundarios del tratamiento (10 ítems hipertensión y 11 ítems diabetes)

8. Usted reconoce cuales son las molestias causadas por el tratamiento para el control de la presión arterial? Menciónelas:

8a. Baja tensión

8b. Boca seca

8c. Depresión (tristeza)

8d. Edema/hinchazón

8e. Estreñimiento

8f. Impotencia sexual

8g. Irritación gástrica (ardor)

8h. Mareo

8i. Tos

8j. Aumento de la frecuencia o cantidad de orina
8. Usted reconoce cuales son las molestias causadas por el tratamiento para el control de la diabetes (azúcar)? Menciónelas: 8ai. Cefalea 8bi. Palpitaciones

8ci. Sudoración

8di. Palidez

8ei. Alteraciones visuales

8fi. Ganas de vomitar y/o vómito

8gi. Mareo

8hi. Temblor

8ii. Sensación de hambre (agonía)

8ji. Hormigueo/entumecimiento

(adormecimiento de extremidades) 8ki. Diarrea

160910 Supervisa los efectos secundarios de la enfermedad (11 ítems)

9. Reconoce las complicaciones de ser diabético y/o hipertenso, de cuales síntomas está usted está pendiente?

9a. Cambio brusco de la agudeza visual

9b. Dolor agudo de los ojos

9c. Hinchazón de los parpados en horas de la mañana

9d. Disminución del volumen urinario

9e. Cansancio fácil

9f. Disminución de pulsos

9g. Perdida de sensibilidad (hormigueo)

9h. Úlceras

9i. Dolor en las piernas durante la marcha

9j. Taquicardia

9k. Intolerancia al ejercicio

160913 Altera las funciones el rol para el cumplimiento terapéutico (4 ítems)

10.Usted:

10a. Durante el día interrumpe las actividades del hogar/trabajo para tomar a tiempo los medicamentos.

10b. Ha modificado labores del trabajo o actividades del hogar para poder cumplir con las recomendaciones dadas por el equipo de salud.

10c. Considera que cumplir con el tratamiento (hacer ejercicio, tomar los medicamentos, asistir a citas) demanda mucho tiempo*.

10d. Los miembros de la familia han modificado las funciones para ayudarle al cumplimiento del tratamiento 


\section{Equilibra actividad y reposo (6 ítems)}

11.Usted:

11a. Duerme entre 6 y 8 horas al día

11b. Al despertar siente que descanso durante la noche

11c. Siente que tiene la energía suficiente para llevar a cabo las actividades diarias

$11 \mathrm{~d}$. Realiza periodos de descanso durante sus labores del trabajo o del hogar

11e. Cuenta con mínimo 30 minutos para tomar cada una de las 3 comidas importantes del día.

11f. En momentos diferentes de las labores del trabajo o del hogar, realiza al menos 1 vez por

día actividades de recreación y descanso (ver TV, caminata, leer, escuchar música chatear en internet, etc.)

*Ítems que califican en sentido contrario

Fuente: elaboración por los autores

\section{Anexo 2}

\section{Construcción, validez de contenido y facial del instrumento para medir la adherencia al tratamiento en hipertensión y diabetes}

Para la construcción del instrumento, se revisó un cuestionario previo que estaba basado en la etiqueta CRE "Conducta Terapéutica: Enfermedad o Lesión" para medir adherencia terapéutica en pacientes hipertensos ${ }^{1}$. Sin embargo, para conseguir una evaluación del cumplimiento al régimen terapéutico más completa e integral, se consideró importante aumentar el número de indicadores e ítems del CRE y que también permitiera medir la adherencia tanto en personas con HTA como DM2. La construcción y restructuración de los ítems estuvo basada en las recomendaciones vigentes de la literatura científica relacionada con el manejo de las dos entidades clínicas, la revisión de manuales de terapéutica y de fisiopatología y la experiencia de los investigadores en el cuidado de poblaciones con estas patologías ( 2 enfermeras y 1 médico).

Al finalizar el proceso de construcción del instrumento, se pasó de una versión de 7 indicadores (37 ítems) ${ }^{1}$ de la etiqueta de resultado CRE conducta terapéutica, a un instrumento de 12 indicadores (97 ítems), que evaluaban el cumplimiento del régimen de medicación, dieta y ejercicio prescrito; además, si se evitaban conductas que potencian la patología, si se equilibraba la actividad y el reposo y si se solicitaban citas y asesoramiento con profesionales cuando se necesitaba; igualmente, si se supervisaban los efectos terapéuticos, efectos secundarios del tratamiento y los cambios de la enfermedad y por último, si se modificaban la funciones del rol para poder cumplir con el régimen terapéutico.

La validez de contenido y facial fue realizada con metodología cualitativa y sin la aplicación de pruebas estadísticas². A continuación, se detalla dicho proceso.

Una vez definidos los indicadores e ítems, se realizó validez de contenido a juicio de 4 expertos (enfermeras con experiencia en el área) para determinar que el cuestionario midiera adherencia terapéutica en HTA y DM basados en la etiqueta NOC "Conducta terapéutica: enfermedad o lesión (1609)", en los lineamientos de las guías de manejo vigentes y en los fundamentos del cuidado de enfermería.

En el proceso los expertos debían establecer si los ítems eran claros, suficientes y comprensibles para cada indicador y estar o no de acuerdo con la propuesta de operacionalización. Posteriormente, se realizó un encuentro para el consenso de expertos donde se analizó indicador por 
indicador e ítem por ítem. A partir del encuentro se hicieron ajustes sugeridos y consensuados por los expertos e investigadores, en cuanto a redacción de los encabezados, número de ítems e indicadores y la operacionalización de algunos indicadores. En general, de manera cualitativa se estableció que había buen acuerdo entre expertos.

Dentro de los ajustes realizados, al indicador "Sigue la dieta prescrita (160919)" se le retiró 2 ítems (¿Cumple con las cantidades recomendadas de los alimentos? y ¿Evita el consumo de enlatados, carnes frías, salsas y paquetes? (chitos, papas...)) por considerarse que estaban contenidos en otros y pasó de 8 a 6 ítems; igualmente al indicador "Supervisa los efectos terapéuticos (160908)" se le retiró 2 ítems (¿Se ha pesado en el último mes? y ¿Sabe si los valores de Glicemia, hemoglobina glicosilada y/o tensión arterial están dentro de los rangos esperados?), por considerarse que uno no era específico para HTA y DM2 y el otro evaluaba conocimiento y no adherencia, es así, que pasó de 5 a 3 ítems.

De otra parte, al indicador "Supervisa los cambios en el estado de enfermedad (160911)", se le retiró los ítems mareo y hormigueo para HTA y náuseas y/o vómito, confusión y dificultad para respirar para DM2; y al indicador "Supervisa efectos secundarios de la enfermedad (160910)", se le retiró los ítems sensación de moscas volantes y/o manchas en los ojos, orina espumosa y eritema (enrojecimiento), por considerarse que no correspondían o eran poco específicos para los procesos de enfermedad.

En este proceso de ajuste, solo se anexaron dos ítems, mareo para el indicador "Supervisa los cambios en el estado de enfermedad (160911)" específicamente para DM2 y aumento de la diuresis para el indicador "Supervisa los efectos secundarios del tratamiento (160909)", específicamente para HTA.

Finalmente, los indicadores: "Solicita cita con profesional sanitario cuando es necesario (160916) con 3 ítems y "Obtiene asesoramiento de un profesional cuando es necesario (160921)" con 4 ítems, fueron agrupados en uno solo con 5 ítems, dado que compartían características similares.

Para concluir, a través del proceso de validación de contenido se pasó de un instrumento de 12 indicadores (97 ítems) a uno de 11 indicadores (85 ítems), para ser medidos de forma dicotómica. En el Anexo 1 se puede apreciar la versión utilizada para la validación de constructo.

En cuanto a la validez facial del instrumento, esta fue evaluada en 30 personas con diagnóstico de HTA y DM2, lo cual permitió evaluar la claridad y comprensión de los ítems por parte de la población de interés. Posteriormente, en una sesión de retroalimentación con los investigadores y las enfermeras encargadas de la aplicación del instrumento, de manera cualitativa se hizo una síntesis de las observaciones y se estableció que en general había alta comprensión por parte de los participantes y que era necesario realizar ajustes mínimos, enfocados principalmente al uso de palabras o términos que fueran fácilmente entendidos por los pacientes.

En consecuencia, para los indicadores 160909, 160910 y 160911, que están relacionados con si la persona supervisa los efectos secundarios del tratamiento, del estado de la enfermedad y complicaciones de la misma, se consideró que las preguntas no eran claras y requerían explicación por parte de las enfermeras encuestadoras, además, se analizó que el preguntar si supervisaban los síntomas o complicaciones uno a uno, se corría el riesgo de condicionar la respuesta por parte de los pacientes. Por tanto, para estos indicadores se acordó preguntar de manera abierta si reconocían los síntomas o complicaciones, en caso afirmativo la persona debía men- 
cionar cuales reconocía. A modo de ejemplo, uno de los ítems quedó de la siguiente forma: “Usted reconoce los síntomas cuándo se le sube la presión arterial? Si la respuesta es sí, ¿Cuáles?

En relación a la operacionalización de los indicadores (once); uno de ellos (160908) se evaluó con tres ítems, cuatro (160904, 160905, 160906 y 160913) se evaluaron con 4 ítems cada uno, dos (160919 y 160920) con 6 ítems cada uno y el indicador fusionado (160916 y 160921) con 5 ítems.

Los indicadores 160909,160910 y 160911 se evaluaron con 21, 11 y 16 ítems respectivamente, los cuales correspondían con los efectos del tratamiento, tanto terapéuticos como secundarios y de la enfermedad hipertensiva y la diabetes; efectos que debían ser vigilados por los pacientes. Debido al gran número y complejidad de algunos de los ítems, en consenso se estableció que si la persona lograba enunciar 4 o más ítems (efectos) en cada uno de los indicadores, obtendría el máximo puntaje (5) y si no enunciaba ninguno el mínimo puntaje (1).

En el indicador 160906 (Evita conductas que potencien la patología), considerando que las preguntas se enfocan hacia los factores de riesgo (consumo de tabaco, alcohol y vivencia de situaciones estresantes), los cuales son aspectos que no favorecen la adherencia; los ítems se redactan en positivo para facilitar la comprensión por parte de los usuarios, pero califican de modo contrario.

\section{Referencias}

1. Páez AN, Caballero LP, López N, Oróstegui M, Orozco LC, Valencia LI. Construct validity of an instrument to assess patient adherence to antihypertensive treatment. Enfermería Global. 2014;13(2):48-57. https://doi.org/10.6018/eglobal.13.2.170111.

2. Carvajal A, Centeno C, Watson R, Martínez M, Sanz Rubiales Á. ¿Cómo validar un instrumento de medida de la salud? Anales del Sistema Sanitario de Navarra. 2011;34(1):6372.http://scielo.isciii.es/scielo.php?script=sci arttext\&pid=S1137-66272011000100007\&ln$\mathrm{g}=\mathrm{es}$. 